\title{
Rare presentation of adenomatoid odontogenic tumor in a pediatric patient: a case report
}

\author{
João Paulo Bonardi ${ }^{1} \cdot$ Fernanda Herrera da Costa ${ }^{2} \cdot$ Ricardo Alves Matheus $^{2}$ • \\ Fabio Augusto Ito $^{2} \cdot$ Cecilia Luiz Pereira-Stabile ${ }^{2}$
}

Received: 19 September 2015 / Accepted: 15 November 2015 /Published online: 20 November 2015

(C) Springer-Verlag Berlin Heidelberg 2015

\begin{abstract}
The adenomatoid odontogenic tumor (AOT) is a painless benign tumor with slow growth, usually asymptomatic. It has three variants: follicular, extrafollicular, and peripheral. In the follicular type, the tumor is associated with an impacted tooth, and maxillary canines are the most frequently affected. Association with primary teeth is very rare. Treatment consists essentially in a total lesion enucleation. The objective of this paper is to present the clinical case of a 7-year-old female patient with an adenomatoid odontogenic tumor associated with the crown of the left lower deciduous canine (73), dislodging it to the mandibular base and consequently shifting and also impacting the permanent lower canine (33). The lesion was treated with careful enucleation, preserving the permanent canine, which then had its eruption path released favoring its migration to an ideal position.
\end{abstract}

Keywords Impacted tooth · Deciduous tooth · Adenomatoid odontogenic tumor $\cdot$ Pediatric Dentistry

\section{Introduction}

One of the first reports on the adenomatoid odontogenic tumor (AOT) was published in 1907, by Dreibladt [1]. Stafne, in

João Paulo Bonardi

joao_bonardi@hotmail.com

1 Department of Surgery and Integrated Clinic, Araçatuba Dental school-UNESP, 1193 José Bonifácio Street, Araçatuba, São Paulo CEP 16015-050, Brazil

2 Department of Oral Medicine and Pediatric Dentistry, State University of Londrina, 540 Pernambuco Street, Londrina, Paraná CEP 86020-120, Brazil
1948, reported three cases and described them as presenting a distinct histology [2]. Statistically, $69 \%$ of the AOTs are diagnosed in the second decade of life, affecting more female patients, in the ratio of 2:1.5 [3] with a predilection for the maxilla [4]. There are three variations of AOTs: follicular (associated with an impacted tooth), extrafollicular (not associated with an impacted tooth), and peripheral. The follicular and extrafollicular types are intraosseous lesions and account for about $96 \%$ of all AOTs of which $71 \%$ are follicular type [5].

Clinically, AOT presents usually as a painless tumor mass with a slow growth rate. Radiographically, it appears as a unilocular radiolucency, suggestive of peripheral bone condensation and the presence of a discrete radiopacity within the lesion. The histopathological characteristics of AOTs are very specific, which prevents somehow confusion with other odontogenic lesions. The microscopic image is characterized by a well-encapsulated structure with a circumscribed intraluminal, thick, and fibrous proliferation of the epithelium. The cells of the epithelium form a solid lump with false ducts, spiral structure, or rosette forms. The ducts may be coated with cylindrical or cubical cells with nuclei polarized away from the lumen. The lumen may be empty or may contain an eosinophilic material of undetermined origin. Mitoses can be seen, but they are not usually numerous; sometimes, a variable amount of calcification and the presence of a hard tissue resembling cementum or dentin are also observed. The pathogenesis of the AOT has not been elucidated and is controversial. Most authors agree that it has an odontogenic origin [5-8]. The most accepted theory is that the tumor develops from the dental lamina [9]. The clinical, radiographic, and microscopic characteristics associated with very few reported recurrences indicate that it consists of a benign lesion. The treatment of choice is the total enucleation of the lesion $[5,10]$. The association between intraosseous 
Fig 1 Computed tomography showing calcification within the lesion (section 86), deciduous canine crown associated with the tumor (section 89), intimate contact between the teeth 73 and 33 (section 92), and proximity between permanent canine and premolar (section 94)

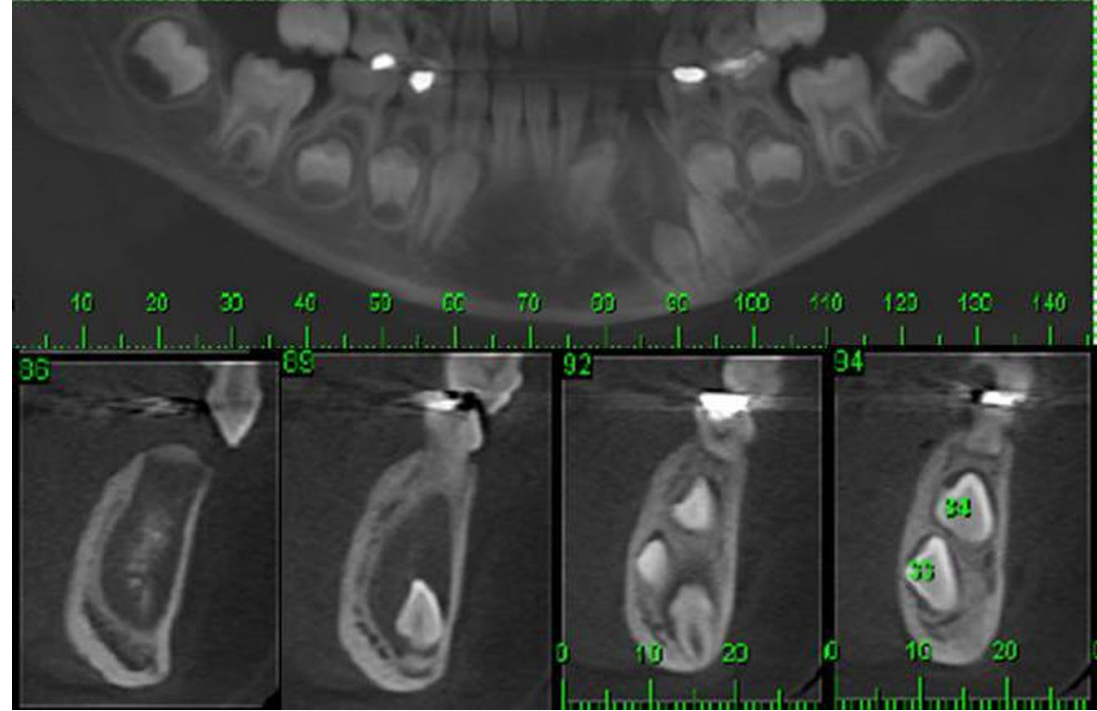

AOT and impacted deciduous teeth is extremely rare [5]. Thus, the aim of this paper is to report a case of this unusual presentation.

\section{Case report}

A 7-year-old female patient attended the oral surgery department at the State University of Londrina dental clinic, referred by a dentist of the public basic health unit (BHU) for evaluation of an intraosseous lesion of the mandible. The lesion was observed during radiographic examination to investigate the absence of tooth 73 . During anamnesis, the mother denied any medical conditions or allergies. At clinical examination, extraoral exam was unremarkable and the only finding intraorally was the absence of tooth 73 . The panoramic radiography showed a unilocular, well-delimited radiolucency with a suggestive image of peripheral opaque bone condensation within the lesion. The image measured approximately $1.0 \times 1.5 \mathrm{~cm}$ and involved the crown of tooth 73 that was dislodged to the base of the mandible and had also displaced tooth 33. The initial diagnostic hypotheses were odontogenic calcifying cyst, follicular AOT, and Pindborg Tumor.

The initial treatment plan was an excisional biopsy through complete surgical enucleation of the lesion. A cone beam computed tomography (CBCT) was performed for better surgical planning and showed an impacted tooth 73 dislodged to the mandible base, with a circumscribed hypodense area around its crown containing mineralization areas suggesting a cyst or benign tumor. The developing tooth 33 had been shifted lower and distal, was impacted and had no space for eruption (Fig. 1).

The patient underwent a surgical procedure under local anesthesia. A Neumann incision, followed by mucoperiosteal

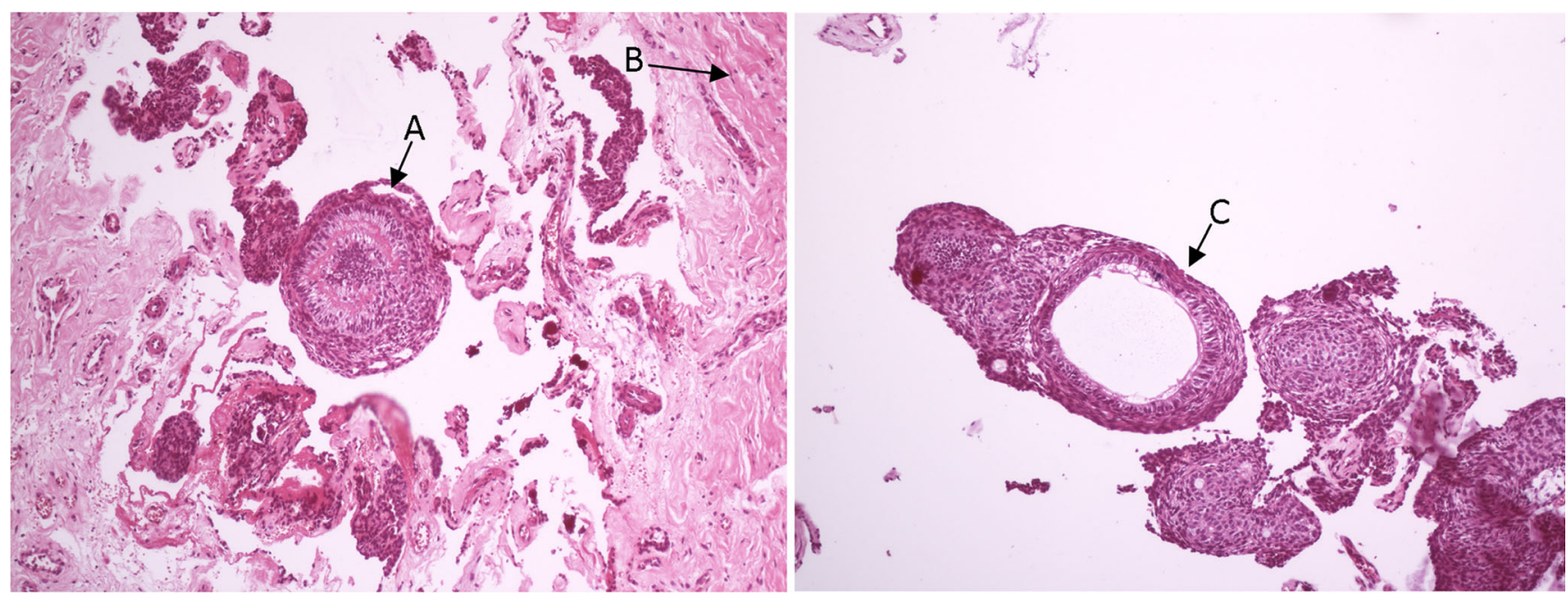

Fig 2 Histologic image, $\times 100$ magnification, HE staining. In $A$, presenting epithelial cells (cuboid cells with polarized nuclei) shaped like rosette and spiral. In $B$, we can observe the presence of a thicker fibrous capsule. In $C$, the cells also form structures resembling ducts 


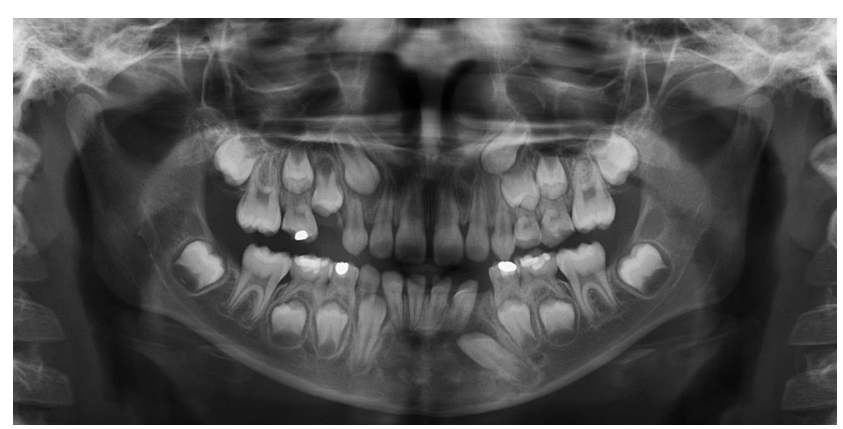

Fig 3 Radiographic image of the favorable position of tooth 33

detachment, and careful osteotomy allowed for the complete removal of the lesion and the affected tooth. The specimen was sent for histopathological analysis, which showed intraluminal proliferation of the epithelium, presenting cuboid cells with polarized nuclei shaped like rosette, spiral, and structures resembling ducts, with a final diagnosis of follicular AOT (Fig. 2).

\section{Discussion}

The clinical case reported differs from the epidemiology presented so far in the literature. In a study conducted in Nigeria and published in 1997, authors reported that the follicular AOT presents higher incidences in males, with a 1.4:1 ratio; the age group most commonly affected was the second decade of life, with a mean age of approximately 15 years for both genders. The maxilla was the most frequent site for the tumor $(76.3 \%)$, and the most affected teeth were the permanent canines $(76.9 \%)$, of which the upper canines presented the highest rate $(83.3 \%)$ [8].

In a review presented by Philippsen and Reichart, in 1998, the epidemiology was similar to the study conducted in Nigeria, except by gender (female), with incidences varying widely with the geographic location [5]. In our clinical case, the patient was a 7-year-old girl, with a mandible lesion related to the deciduous canine, which is a very unusual presentation. Until the 1990s, only two cases of AOT associated with deciduous impacted teeth had been reported [4], and no other reports were found in the literature during review for this paper, which confirms the extreme rarity of the case.

With the initial radiographic examination, it could be deduced that the cause of the deciduous tooth impaction was a lesion that involved the deciduous tooth's crown and consequently caused the impaction of the permanent successor. Initial treatment for all the possible diagnosed hypotheses was, essentially, the same (enucleation). So, in an attempt to provide the best treatment for the permanent tooth, a cone beam computed tomography was requested, to collect more information about the actual dimension of lesion, the accurate position of the permanent tooth, and the relationship between lesion, deciduous tooth, and permanent tooth. With all this information, it was possible to plan a less traumatic surgical procedure to the permanent tooth, providing it with some space for proper irruption. The patient has recovered well since the initial surgery and has been followed by the oral surgery service for 12 months. At a 12-month postoperative radiographic examination, it was possible to note the migration of tooth 33 to a more favorable position (Fig. 3). If necessary, the next step of treatment may involve exposure and bonding of an orthodontic apparatus for traction of the permanent canine.

Compliance with ethical standards The manuscript does not contain clinical studies or patient data.

Conflict of interest The authors declare that they have no competing interests.

\section{References}

1. Philipsen HP, Reichart PA, Zhang KH, Nikai H, Yu QX (1991) Adenomatoid odontogenic tumor: biologic profile based on 499 cases. J Oral Pathol Med 20(4):149-158

2. Stafne EC (1948) Epithelial tumors associated with developmental cysts of the maxilla; a report of three cases. Oral Surg Oral Med Oral Pathol 1(10):887-894

3. Philipsen HP, Reichart PA, Nikai H (1997) The adenomatoid odontogenic tumour (AOT): an update. Oral Med Pathol 2:55-60

4. Toida M, Hyodo I, Okuda T, Tatematsu N (1990) Adenomatoid odontogenic tumor: report of two cases and survey of 126 cases in Japan. J Oral Maxillofac Surg 48(4):404-408

5. Philipsen HP, Reichart PA (1999) Adenomatoid odontogenic tumour: facts and figures. Oral Oncol 35(2):125-131

6. Abrams AM, Melrose RJ, Howell FV (1968) Adenoameloblastoma: a clinicopathologic study of ten new cases. Cancer 22:175-85

7. Giansanti JS, Someren A, Waldron CA (1970) Odontogenic adenomatoid tumor (adenoameloblastoma) Survey of 111 cases. Oral Surg Oral Med Oral Pathol 30(1):69-88

8. Arotiba GT, Arotiba JT, Olaitan AA, Ajayi OF (1997) The adenomatoid odontogenic tumor: an analysis of 57 cases in a Black African population. J Oral Maxillofac Surg 55(2):146-148

9. Philipsen HP, Samman N, Ormiston IW, Wu PC, Reichart PA (1992) Variants of the adenomatoid odontogenic tumour with a note on tumour origin. J Oral Pathol Med 21(8):348-352

10. Courtney RM, Kerr DA (1975) The odontogenic adenomatoid tumor a comprehensive study of twenty new cases. Oral Surg Oral Med Oral Pathol 39(3):424-435 